hep-th/0604219

ITP-UH-08/06

\title{
Moduli-Space Dynamics of Noncommutative Abelian Sigma-Model Solitons
}

\author{
Michael Klawunn, Olaf Lechtenfeld and Stefan Petersen \\ Institut für Theoretische Physik, Universität Hannover \\ Appelstraße 2, 30167 Hannover, Germany \\ Email: klawunn, lechtenf, petersen @itp.uni-hannover.de
}

\begin{abstract}
In the noncommutative (Moyal) plane, we relate exact $\mathrm{U}(1)$ sigma-model solitons to generic scalar-field solitons for an infinitely stiff potential. The static $k$-lump moduli space $\mathbb{C}^{k} / S_{k}$ features a natural Kähler metric induced from an embedding Grassmannian. The moduli-space dynamics is blind against adding a WZW-like term to the sigma-model action and thus also applies to the integrable U(1) Ward model. For the latter's two-soliton motion we compare the exact field configurations with their supposed moduli-space approximations. Surprisingly, the two do not match, which questions the adiabatic method for noncommutative solitons.
\end{abstract}




\section{Introduction and summary}

Field theories with a nontrivial vacuum structure often feature static localized finite-energy solutions. Such lumps can be boosted to single solitons moving with constant velocity. The scattering of these solitons off one another is, however, usually accessible only numerically. ${ }^{1}$ Alternatively, a qualitative understanding of soliton scattering can be achieved for small relative velocity via the adiabatic or moduli-space dynamics invented by Manton 1, 2. This approach approximates the exact $k$-soliton scattering configuration by a time sequence of static $k$-lump solutions. Thereby one introduces a time dependence for the latter's moduli $\alpha_{i}$, which is determined by extremizing the action on the moduli space $\mathcal{M}_{k}$. Being a functional of finitely many moduli $\alpha_{i}(t)$, this action describes the motion of a point particle in $\mathcal{M}_{k}$, equipped with a metric $g_{i j}(\alpha)$ and a magnetic field $A_{i}(\alpha)$. Hence, the motion of $k$ slowly scattering solitons is well described by a geodesic trajectory in $\mathcal{M}_{k}$, possibly with magnetic forcing. Since among the moduli are the spatial locations of the individual quasi-static lumps, (a projection of) the geodesic in $\mathcal{M}_{k}$ may be viewed as trajectories of the various lumps in the common ambient space.

In order to test the validity of the adiabatic method, one would need to apply it to an integrable model, where exact multi-soliton solutions are available for comparison. Yet, such theories are rare in two or more spatial dimensions, which are required for interesting trajectories. A prime example is the nonlinear sigma model for some group $\mathcal{G}$ in $1+2$ dimensions. By adding a (necessarily Lorentz-breaking) WZW-like term with an arbitrary coefficient, one generates a one-parameter family of extended sigma models. Their common static configuration space is well known to contain multiple lumps (one-unitons), which are based on hermitian projectors and thus sit in certain Grassmannians [3, 4, 5]. For a particular strength of the WZW-like term, one obtains the Ward model, which is integrable [6, 7, 8]. Recently, the adiabatic approach was tested in this model for $\mathcal{G}=\mathrm{SU}(2)[9]$.

It is rewarding to generalize the above set of ideas to field theories on noncommutative spaces. Such field theories offer not only smooth deformations of well known soliton solutions but entirely new types of noncommutative solitons (for lectures on the subject see [10]). This property is most prominent when the commutative limit yields a free theory, because the soliton configurations are then forced to become singular in this limit. A case in point is the abelian sigma model, i.e. choosing $\mathcal{G}=\mathrm{U}(1)$, on a noncommutative plane with ordinary time. It has the virtue that its static $k$-lump solutions take a very simple form and depend exclusively on the $k$ complex location moduli [11, 12]. Furthermore, its extension à la Ward is again integrable 13. Therefore, the family of extended $\mathrm{U}(1)$ sigma models seems ideally suited to try out the adiabatic method in the noncommutative realm, and this is what we do in the present paper. For definiteness, we work with the standard Moyal deformation, labelled by a positive constant parameter $\theta$. The Moyal-Weyl map is employed to pass from the star product to the operator realization on the harmonic-oscillator Fock space $\mathcal{H}$.

The sigma-model constraint can be implemented in unconstrained (multi-component) scalar field models by choosing an appropriate potential and performing an infinite-stiffness limit. Therefore, the soliton analysis for generic noncommutative field theories in $1+2$ dimensions [10, 14, 15, 16, 17, 18, 19] applies to noncommutative sigma models (without WZW-like term) as well. In fact, it yields the exact static multi-lumps on the Moyal plane for any value of $\theta$, as we shall review in section 2 below. More precisely, the $k$-lump moduli space $\mathcal{M}_{k}$ is parametrized by all collections of $k$ harmonic-oscillator coherent states and is a $k$-dimensional complex submanifold of the

\footnotetext{
${ }^{1}$ Exceptions are integrable theories, which allow for analytic multi-soliton configurations and an exact S-matrix.
} 
Grassmannian $\operatorname{Gr}(k, \mathcal{H})$. Section 3 computes the full moduli-space action for the noncommutative $\mathrm{U}(1)$ extended sigma model. For reasons to be explained, the result turns out to agree with the $\theta$-independent part of the moduli-space action for the generic scalar field theory, irrespective of the WZW-like term. More concretely, $\mathcal{M}_{k}$ has a natural Kähler structure, with the Kähler potential being given by the determinant of the matrix of coherent-state overlaps [17. There is no magnetic background field. We briefly discuss the properties of the moduli-space metric and its limits for coinciding lumps.

The moduli-space scattering trajectories for two solitons are investigated in section 4 and exhibit scattering angles between 0 and $\frac{\pi}{2}$. Finally, section 5 compares with the time-dependent solutions of the field theory family, in particular with the exact multi-soliton configurations of the U(1) Ward model. Barring some miracle, the latter features only solitonic no-scattering or bound-state solutions, which we display. It appears that the adiabatic approximation fails in this integrable case, possibly due to its inability to sense the WZW-like action term. A numerical investigation, also away from the integrable case, could help settle this issue. Finally, we remark that our considerations are purely classical and likely to be modified by quantum corrections.

\section{Noncommutative static lumps in scalar field and sigma models}

We begin with a rather generic action of a real scalar field $\phi$ living on the Moyal plane with coordinates $(z, \bar{z})$ and depending on time $t$,

$$
S_{\theta}=\int \mathrm{d} t \mathrm{~d}^{2} z\left[\frac{1}{2} \dot{\phi}^{2}-\partial_{z} \phi \partial_{\bar{z}} \phi-V_{\star}(\phi)\right],
$$

where the subscript on the potential signifies star-product multiplication based on

$$
z \star \bar{z}-\bar{z} \star z=2 \theta
$$

We further specify

$$
V(\phi) \geq 0 \quad, \quad V\left(\phi_{0}\right)=0 \quad \text { and } \quad V^{\prime}(\phi)=v \prod_{i}\left(\phi-\phi_{i}\right)
$$

Static classical configurations $\phi_{\mathrm{cl}}$ extremize the energy functional

$$
E_{\theta}=\int \mathrm{d}^{2} z\left[\partial_{z} \phi \partial_{\bar{z}} \phi+V_{\star}(\phi)\right]
$$

which for large values of $\theta$ is dominated by the potential term, because $z=O(\sqrt{\theta})$. Expanding around $\theta=\infty$, one obtains [17]

$$
\phi_{\mathrm{cl}}=\widehat{\phi}+\frac{1}{\theta} \tilde{\phi}+\ldots \quad \text { with } \quad \widehat{\phi}=\sum_{i} \phi_{i} P_{i}
$$

where $\left\{P_{i}\right\}$ is an orthogonal resolution of the star-algebra identity,

$$
P_{i} \star P_{j}=\delta_{i j} P_{j} \quad \text { and } \quad \sum_{i} P_{i}=\mathbb{1} .
$$


We also introduce the rank $k_{i}$ of $P_{i}$ via

$$
\int \frac{\mathrm{d}^{2} z}{2 \pi \theta} P_{i}=k_{i} \in \mathbb{N}_{0}
$$

Since $\tilde{\phi}$ and all further terms in the expansion are determined by $\widehat{\phi}$, any classical solution is fixed by an assignment of projectors $P_{i}$ to the extrema $\phi_{i}$ of the potential. Restricting ourselves to stable solutions, we always associate the zero projector (which is admissible) to the local maxima of $V$. Please note that the collection $\left\{P_{i}\right\}$ appearing in (2.5) must be complete. The expansion of the classical energy reads

$$
E_{\theta}\left[\phi_{\mathrm{cl}}\right]=\theta E_{0}+E_{1}+\frac{1}{\theta} E_{2}+\ldots \quad \text { with } \quad E_{0}=2 \pi \sum_{i} k_{i} V\left(\phi_{i}\right)
$$

and

$$
E_{1}=\int \mathrm{d}^{2} z \partial_{z} \widehat{\phi} \partial_{\bar{z}} \widehat{\phi}=\sum_{i j} \phi_{i} \phi_{j} \int \mathrm{d}^{2} z \partial_{z} P_{i} \partial_{\bar{z}} P_{j}
$$

Any complete collection $\left\{P_{i}\right\}$ extremizes $E_{\theta}$ at leading order in $\theta$. Beyond this, however, $E_{1}$ lifts this infinite degeneracy: its extremization selects a finite-dimensional class of identity resolutions.

In [17, an asymmetric double-well potential was chosen, with local minima $V(0)=0$ and $V(\lambda)>0$. The authors assigned

$$
\phi=\lambda \quad \leftrightarrow \quad P \quad \text { and } \quad \phi=0 \quad \leftrightarrow \quad \mathbb{1}-P
$$

which led to

$$
\widehat{\phi}=\lambda P \quad \text { as well as } \quad E_{0}=2 \pi k V(\lambda) \quad \text { and } \quad E_{1}=\lambda^{2} \int \mathrm{d}^{2} z\left|\partial_{z} P\right|^{2} .
$$

Presently, we consider instead the double-well potential

$$
V(\phi)=\beta\left(\phi^{2}-1\right)^{2} \quad \longrightarrow \quad V^{\prime}(\phi)=4 \beta(\phi+1) \phi(\phi-1)
$$

and associate

$$
\phi=-1 \quad \leftrightarrow \quad P \quad \text { and } \quad \phi=+1 \quad \leftrightarrow \quad \mathbb{1}-P
$$

which implies

$$
\widehat{\phi}=\mathbb{1}-2 P \quad \text { as well as } \quad E_{0}=0 \quad \text { and } \quad E_{1}=4 \int \mathrm{d}^{2} z\left|\partial_{z} P\right|^{2}
$$

It is easy to see that all higher corrections, i.e. $\tilde{\phi}, E_{2}$ etc., come with negative powers of $\beta$. Therefore, we have the exact result

$$
\phi_{\mathrm{cl}} \rightarrow \widehat{\phi} \quad \text { and } \quad E_{\theta}\left[\phi_{\mathrm{cl}}\right] \rightarrow E_{1}
$$

in the limit of infinite stiffness, $\beta \rightarrow \infty$, and there is no effective potential on the moduli space. This limit nails the value of $\phi$ to $-1($ in $\operatorname{im} P$ ) or to +1 (in $\operatorname{ker} P$ ) and makes the classical configuration idempotent, i.e. $\widehat{\phi}_{\star}^{2}=\mathbb{1}$.

Idempotent fields also appear in nonlinear sigma models, where they define Grassmannian submanifolds of the group $\mathcal{G}$ via $P=\frac{1}{2}(1-\phi)$. The simplest case occurs for $\mathcal{G}=\mathrm{U}(1)$, i.e. for complex 
unimodular $\phi$, and becomes interacting when being Moyal deformed. ${ }^{2}$ Its two Grassmannian submanifolds correspond precisely to the two idempotent values above, namely $\phi= \pm 1$. Hence, if we extend our double-well model to a Mexican-hat model for complex $\phi$, the stiff limit will yield the constraint $|\phi|_{\star}^{2}=\mathbb{1}$ defining the $\mathrm{U}_{\star}(1)$ sigma model, and our trial configurations $\widehat{\phi}$ for $P$ of rank $k$ parametrize precisely the Grassmannian $\operatorname{Gr}(k, \mathcal{H})$. The only modification owed to the extension is a factor of two in the energy functional. For $k<\infty$, the latter can be manipulated to

$$
E_{1}=8 \int \mathrm{d}^{2} z\left|\partial_{z} P\right|^{2}=8 \pi k+16 \int \mathrm{d}^{2} z\left|(\mathbb{1}-P) \star \partial_{\bar{z}} P\right|^{2} \geq 8 \pi k
$$

revealing a Bogomolnyi bound..$^{3}$ The saturation $E_{\mathrm{BPS}}=8 \pi k$ is reached when

$$
(\mathbb{1}-P) \star \partial_{\bar{z}} P=0 \quad, \quad \text { i.e. } \partial_{\bar{z}}: \operatorname{im} P \hookrightarrow \operatorname{im} P
$$

Hence, the static classical configurations $\widehat{\phi}=\mathbb{1}-2 P$ extremizing $E_{1}$ are given by projectors stable under the $\partial_{\bar{z}}$ action, and their moduli space $\mathcal{M}_{k}$ for rank $k$ describes static $k$-lump solutions of the $\mathrm{U}_{\star}(1)$ sigma model in the Moyal plane.

In order to find an explicit parametrization of $\mathcal{M}_{k}$, we pass from the star-product to the operator formulation,

$$
z \rightarrow \sqrt{2 \theta} a \quad, \quad \bar{z} \rightarrow \sqrt{2 \theta} a^{\dagger} \quad \text { hence } \quad \sqrt{2 \theta} \partial_{z} \rightarrow-\left[a^{\dagger}, .\right] \quad, \quad \sqrt{2 \theta} \partial_{\bar{z}} \rightarrow[a, .]
$$

with

$$
\left[a, a^{\dagger}\right]=\mathbb{1} \quad \text { and } \quad \int \mathrm{d}^{2} z \ldots=2 \pi \theta \operatorname{tr}_{\mathcal{H}} \ldots,
$$

justifying the definition (2.7). The Fock space $\mathcal{H}$ representing this Heisenberg algebra is spanned by the basis

$$
|n\rangle=\frac{1}{\sqrt{n !}}\left(a^{\dagger}\right)^{n}|0\rangle \quad \text { with } \quad n \in \mathbb{N}_{0} \quad \text { and } \quad a|0\rangle=0 .
$$

Any rank- $k$ projector in $\mathcal{H}$ can be decomposed as

$$
P=|T\rangle \frac{1}{\langle T \mid T\rangle}\langle T| \quad \text { with } \quad|T\rangle=\left(\left|T_{1}\right\rangle,\left|T_{2}\right\rangle, \ldots,\left|T_{k}\right\rangle\right)
$$

where the (not necessarily orthonormal) states $\left|T_{i}\right\rangle$ span the image of $P$. The BPS equation (2.17) now reads

$$
(\mathbb{1}-P) a P=0 \quad \Longleftrightarrow \quad a|T\rangle=|T\rangle \Gamma \quad \text { for some } k \times k \text { matrix } \Gamma \quad
$$

Generically, the freedom of basis change in $\mathrm{im} P$ can be used to diagonalize

$$
\Gamma \rightarrow \operatorname{diag}\left(\alpha_{1}, \alpha_{2}, \ldots, \alpha_{k}\right) \quad \text { with } \quad \alpha_{i} \in \mathbb{C}
$$

so that we have

$$
a\left|T_{i}\right\rangle=\left|T_{i}\right\rangle \alpha_{i} \quad \Longrightarrow \quad\left|T_{i}\right\rangle=\left|\alpha_{i}\right\rangle \equiv \mathrm{e}^{\alpha_{i} a^{\dagger}}|0\rangle
$$

\footnotetext{
${ }^{2}$ The action will be given in the following section.

${ }^{3}$ A finite $k$ also agrees with the topological charge $Q$ of the respective Grassmannian. Negative values of $Q$ are produced by the flip $P \longleftrightarrow \mathbb{1}-P$ and correspond to anti-solitons.
} 
revealing the key role of coherent states. We note that our solution depends on $k$ complex moduli parameters. The ensueing BPS projector

$$
P_{\alpha}=\sum_{i, j=1}^{k}\left|\alpha_{i}\right\rangle(\langle\alpha . \mid \alpha .\rangle)_{i j}^{-1}\left\langle\alpha_{j}\right|
$$

generates a superposition of $k$ Gaussian lumps in the Moyal plane. Besides the (inessential) choice of normalizations, only a residual permutation freedom remains in the solution $|T\rangle=|\alpha\rangle:=$ $\left(\left|\alpha_{1}\right\rangle,\left|\alpha_{2}\right\rangle, \ldots,\left|\alpha_{k}\right\rangle\right)$. This corresponds to a relabelling of the lumps and emphasizes their bosonic character. The general situation allows for coinciding values of some $\alpha_{i} \rightarrow \alpha$, which leaves $\Gamma$ in Jordan form. A Jordan block of size $r$ yields a sub-basis $\left\{|\alpha\rangle, a^{\dagger}|\alpha\rangle, \ldots,\left(a^{\dagger}\right)^{r-1}|\alpha\rangle\right\}$, whose span is obviously invariant under the action of $a$. Clearly, the 'fusion' of lumps smoothly produces lumps of higher 'weight'. These observations determine the moduli space $\mathcal{M}_{k}$ as the $k$-th symmetrized power of the complex plane, i.e.

$$
\mathcal{M}_{k}=\mathbb{C}^{k} / S_{k} \cong \mathbb{C}^{k}
$$

which is a smooth Kähler manifold despite the coordinate singularities at the coincidence loci [17.

\section{Soliton moduli-space action for a family of abelian sigma models}

We formulate the action for the extended noncommutative abelian sigma model in star-product language. For the group-valued field

$$
\phi \in \mathrm{U}_{\star}(1) \quad, \quad \text { i.e. } \phi \star \phi^{\dagger}=\mathbb{1}=\phi^{\dagger} \star \phi,
$$

we define the antihermitian composite flat gauge connection

$$
J:=\phi^{\dagger} \star \mathrm{d} \phi \quad \longrightarrow \quad F \equiv \mathrm{d} J+J \wedge J=0
$$

The action is a sum

$$
S_{\gamma}=S_{2}+\gamma S_{3} \quad \text { for } \quad \gamma \in[0,1],
$$

where standard sigma-model term

$$
S_{2}=\frac{1}{2} \int J \wedge * J
$$

is formulated with a wedge product based on the star product and the Hodge star depending on the $1+2$ dimensional Minkowski metric $\eta$. The WZW-like term

$$
S_{3}=-\frac{1}{3} \int_{0}^{1} \int \wedge \widetilde{J} \wedge \widetilde{J} \wedge \widetilde{J}
$$

is an integral over $\mathbb{R}_{\theta}^{1,2} \times[0,1]$, with the extension $\widetilde{J}$ interpolating along the interval $[0,1]$ between $\widetilde{J}=0$ and $\widetilde{J}=J$. Furthermore, there appears the Lorentz-breaking constant one-form

$$
V=\mathrm{d} x \quad \text { for } \quad z=x+\mathrm{i} y \quad
$$

By varying $\gamma$, we get a family of actions connecting the ordinary (non-chiral) sigma model (at $\gamma=0$ ) to the (chiral) Ward model (at $\gamma=1$ ), both based on $\mathrm{U}_{\star}(1)$. 
Introducing coordinates $\left(x^{\mu}\right)=\left(x^{0}, x^{1}, x^{2}\right)=(t, x, y)$ and subjecting the field to a general coordinate transformation $\delta \phi=\xi^{\mu}(x) \partial_{\mu} \phi$, the action changes by

$$
\delta S_{\gamma}=\int\left\{\partial^{(\mu} \xi^{\nu)} T_{\mu \nu} \mathrm{d}^{2} z \mathrm{~d} t+\gamma V(\xi) J \wedge J \wedge J\right\}
$$

which, writing $J=J_{\mu} \mathrm{d} x^{\mu}$, yields the standard energy-momentum tensor

$$
T_{\mu \nu}=J_{\mu} \star J_{\nu}-\frac{1}{2} \eta_{\mu \nu} \eta^{\rho \sigma} J_{\rho} \star J_{\sigma}
$$

The Lorentz group $\mathrm{SO}(1,2)$ is broken to the $y$-boosts by the choice of $V$ and, independently, to the $x y$-rotations by the Moyal deformation, leaving nothing. Since $y$ - and $t$-translations are unbroken ${ }^{4}$ the energy functional

$$
E=\frac{1}{2} \int \mathrm{d}^{2} z\left\{\partial_{t} \phi^{\dagger} \partial_{t} \phi+\partial_{x} \phi^{\dagger} \partial_{x} \phi+\partial_{y} \phi^{\dagger} \partial_{y} \phi\right\}
$$

is conserved for all values of $\gamma$.

Finally, the equation of motion reads

$$
\begin{aligned}
0 & =\left(\eta^{\mu \nu}+\gamma V_{\rho} \varepsilon^{\rho \mu \nu}\right) \partial_{\mu}\left(\phi^{\dagger} \star \partial_{\nu} \phi\right) \\
& =\partial_{x}\left(\phi^{\dagger} \star \partial_{x} \phi\right)+(1-\gamma) \partial_{y}\left(\phi^{\dagger} \star \partial_{y} \phi\right)-(1-\gamma) \partial_{t}\left(\phi^{\dagger} \star \partial_{t} \phi\right)+\gamma \partial_{y-t}\left(\phi^{\dagger} \star \partial_{y+t} \phi\right)
\end{aligned}
$$

with the Minkowski metric $\left(\eta_{\mu \nu}\right)=\operatorname{diag}(-1,+1,+1)$ and the Levi-Civita tensor $\varepsilon$, where $\varepsilon^{012}=1$.

For the adiabatic approximation, we need to find the static multi-lump solutions $\widehat{\phi}(x, y)$. Since static configurations do not contribute to $S_{3}$, the energy $E$ reduces to $E_{1}$ in (2.9). Hence, the moduli space of static multi-lumps is the same for all $\gamma$, namely $\mathcal{M}_{k}$ as derived in previous section. Abbreviating the $k$ complex moduli by $\alpha$, we denote the static $k$-lump solution by $\widehat{\phi}(z, \bar{z} ; \alpha)$. To extract the time dependence in the action, we rewrite the latter as

$$
S_{\gamma}[\phi]=\int \mathrm{d} t \mathrm{~d}^{2} z\left[\frac{1}{2} \dot{\phi}^{2}+C_{\star}\left(\phi, \phi^{\prime}\right) \dot{\phi}-W_{\star}\left(\phi, \phi^{\prime}\right)\right] \quad \text { with } \quad \phi^{\prime} \equiv\left(\partial_{z} \phi, \partial_{\bar{z}} \phi\right)
$$

Manton posits that slow soliton motion follows a geodesic of the static moduli space $\mathcal{M}_{k}$, i.e.

$$
\widehat{\phi}(t, z, \bar{z}) \approx \widehat{\phi}(z, \bar{z} ; \alpha(t))=: \phi_{\alpha}
$$

thus replacing dynamics for $\widehat{\phi}(t, z, \bar{z})$ with dynamics for $\alpha(t)$. We are instructed to compute

$$
\begin{aligned}
S_{\text {mod }}[\alpha] & :=S\left[\phi_{\alpha}\right] \\
& =\int \mathrm{d} t\left[\frac{1}{2}\left\{\int\left(\partial_{\alpha} \phi_{\alpha}\right)^{2}\right\} \dot{\alpha}^{2}+\left\{\int C_{\star}\left(\phi_{\alpha}, \phi_{\alpha}^{\prime}\right) \partial_{\alpha} \phi_{\alpha}\right\} \dot{\alpha}-\int W_{\star}\left(\phi_{\alpha}, \phi_{\alpha}^{\prime}\right)\right] \\
& =: \int \mathrm{d} t\left[\frac{1}{2} g_{\alpha \alpha}(\alpha) \dot{\alpha}^{2}+A_{\alpha}(\alpha) \dot{\alpha}-U(\alpha)\right]
\end{aligned}
$$

and read off the metric $g$, magnetic field $F=\mathrm{d} A$ and potential $U$ on the moduli space.

\footnotetext{
${ }^{4}$ Under $x$-translations, $\delta S_{\gamma} \sim \gamma \int J \wedge J \wedge J=24 \pi^{2} \gamma n$ with $n \in \pi_{3}(\mathcal{G})=\mathbb{Z}$ mostly. However, $n=0$ for $\phi \in \operatorname{Gr}(k, \mathcal{H})$.
} 
To implement this program for the extended deformed abelian sigma model, we return to the operator formulation. Putting $\alpha_{i} \rightarrow \alpha_{i}(t)$ introduces $t$-dependence into

$$
|\alpha\rangle=\left(\left|\alpha_{1}\right\rangle, \ldots,\left|\alpha_{k}\right\rangle\right) \quad \rightarrow \quad P_{\alpha}=|\alpha\rangle \frac{1}{\langle\alpha \mid \alpha\rangle}\langle\alpha| \quad \rightarrow \quad \phi_{\alpha}=\mathbb{1}-2 P_{\alpha} \quad \rightarrow \quad \widehat{J}=2\left[P_{\alpha}, \mathrm{d} P_{\alpha}\right] .
$$

Inserting the obtained $\widehat{J}(\alpha, \dot{\alpha})$ into the action (3.3) we make two important observations. Firstly,

$$
S_{3}\left[\phi_{\alpha}\right] \sim \int \mathrm{d} t \partial_{t} \operatorname{tr}_{\mathcal{H}}\left[\left(a+a^{\dagger}\right) P_{\alpha}\right]=\int \mathrm{d} t\left\{\partial_{\alpha_{i}} \operatorname{tr}_{\mathcal{H}}\left[\left(a+a^{\dagger}\right) P_{\alpha}\right]\right\} \dot{\alpha}_{i}+\text { c.c. }
$$

which reveals the magnetic potential to be exact, $A_{i}=\partial_{\alpha_{i}} \Omega$. Thus, magnetic forcing is absent, ${ }^{5}$ and $S_{\text {mod }}$ is independent of $\gamma$. Secondly, we get

$$
S_{2}\left[\phi_{\alpha}\right]=\int \mathrm{d} t \operatorname{tr}_{\mathcal{H}}\left[\pi \theta\left|\dot{\phi}_{\alpha}\right|^{2}-2 \pi\left|\left[a, \phi_{\alpha}\right]\right|^{2}\right]=\int \mathrm{d} t\left[4 \pi \theta \operatorname{tr}_{\mathcal{H}} \dot{P}_{\alpha}^{2}-E\left[\phi_{\alpha}\right]\right] .
$$

Because $E\left[\phi_{\alpha}\right]=8 \pi k$ is constant inside $\mathcal{M}_{k}$, the second term yields an irrelevant constant potential $U$ and can be dropped.

As a result, $S_{\text {mod }}$ reduces to the kinetic part of $S_{2}\left[\phi_{\alpha}\right]$, which simplifies to

$$
\begin{aligned}
S_{\text {mod }}=4 \pi \theta \int \mathrm{d} t \operatorname{tr}_{\mathcal{H}} \dot{P}_{\alpha}^{2} & =8 \pi \theta \int \mathrm{d} t \operatorname{tr}_{\mathcal{H}}\left(\mathbb{1}-P_{\alpha}\right)|\dot{\alpha}\rangle\langle\alpha \mid \alpha\rangle^{-1}\langle\dot{\alpha}| \\
& =8 \pi \theta \int \mathrm{d} t \operatorname{tr}_{k}\langle\alpha \mid \alpha\rangle^{-1}\left\langle\dot{\alpha}\left|\mathbb{1}-P_{\alpha}\right| \dot{\alpha}\right\rangle=: \int \mathrm{d} t \sum_{i, j=1}^{k} g_{\bar{\imath} j} \dot{\bar{\alpha}}_{i} \dot{\alpha}_{j},
\end{aligned}
$$

where

$$
|\dot{\alpha}\rangle \equiv \partial_{t}|\alpha\rangle=a^{\dagger}|\alpha\rangle \dot{\Gamma} \quad \text { with } \quad \Gamma=\operatorname{diag}\left(\left\{\alpha_{i}\right\}\right) .
$$

Hence, abbreviating $\partial_{\alpha_{j}} \equiv \partial_{j}$ and $\partial_{\bar{\imath}} \equiv \partial_{\bar{\alpha}_{i}}$, the metric on $\mathcal{M}_{k}$ is given by

$$
g_{\bar{\imath} j}=8 \pi \theta \operatorname{tr}_{\mathcal{H}} \partial_{\bar{\imath}} P_{\alpha} \partial_{j} P_{\alpha}=8 \pi \theta \operatorname{tr}_{k}\langle\alpha \mid \alpha\rangle^{-1} \partial_{\bar{\imath}} \Gamma^{\dagger}\left\langle\alpha\left|a\left(\mathbb{1}-P_{\alpha}\right) a^{\dagger}\right| \alpha\right\rangle \partial_{j} \Gamma .
$$

With the shorthand

$$
M=\left(M_{i j}\right):=\left(\langle\alpha \mid \alpha\rangle_{i j}\right)=\left(\left\langle\alpha_{i} \mid \alpha_{j}\right\rangle\right)=\left(\mathrm{e}^{\bar{\alpha}_{i} \alpha_{j}}\right)
$$

one computes

$$
\begin{aligned}
S_{\text {mod }} & =8 \pi \theta \int \mathrm{d} t \operatorname{tr}_{k}\langle\alpha \mid \alpha\rangle^{-1} \dot{\Gamma}^{\dagger}\left\langle\alpha\left|a\left(\mathbb{1}-P_{\alpha}\right) a^{\dagger}\right| \alpha\right\rangle \dot{\Gamma} \\
& =8 \pi \theta \int \mathrm{d} t \sum_{i, j=1}^{k} M_{j i}^{-1} \dot{\bar{\alpha}}_{i}\left\langle\alpha_{i}\left|a\left(\mathbb{1}-P_{\alpha}\right) a^{\dagger}\right| \alpha_{j}\right\rangle \dot{\alpha}_{j} \\
& =8 \pi \theta \int \mathrm{d} t \sum_{i, j=1}^{k} M_{j i}^{-1}\left\{M_{i j}\left(1+\bar{\alpha}_{i} \alpha_{j}\right)-\sum_{m, n=1}^{k} M_{i m} \alpha_{m} M_{m n}^{-1} \bar{\alpha}_{n} M_{n j}\right\} \dot{\bar{\alpha}}_{i} \dot{\alpha}_{j} \\
& =8 \pi \theta \int \mathrm{d} t \sum_{i, j=1}^{k} M_{j i}^{-1}\left\{M+\Gamma^{\dagger} M \Gamma-M \Gamma M^{-1} \Gamma^{\dagger} M\right\}_{i j} \dot{\bar{\alpha}}_{i} \dot{\alpha}_{j} \quad,
\end{aligned}
$$

\footnotetext{
${ }^{5}$ The holonomy of $A=A_{\alpha} \mathrm{d} \alpha$ may yet be nontrivial.
} 
which reveals the hermitian metric $\left(g_{\bar{\imath} j}\right)$ on $\mathcal{M}_{k}$. Using the identities

$$
\partial_{j} M=\Gamma^{\dagger} M \partial_{j} \Gamma \quad \text { and } \quad \partial_{\bar{\imath}} M=\partial_{\bar{\imath}} \Gamma^{\dagger} M \Gamma \quad,
$$

it is straightforward to check that this metric is indeed Kähler and derives from the Kähler potential

$$
K=8 \pi \theta \ln \operatorname{det} M=8 \pi \theta \ln \operatorname{det}\left(\left\langle\alpha_{i} \mid \alpha_{j}\right\rangle\right)=8 \pi \theta \ln \operatorname{det}\left(\mathrm{e}^{\bar{\alpha}_{i} \alpha_{j}}\right) .
$$

This result agrees with the geometric intuition: up to the prefactor of $8 \pi \theta$, the metric $g_{\bar{\imath} j}=\partial_{\bar{\imath}} \partial_{j} K$ is the natural one on the Grassmannian $\operatorname{Gr}(k, \mathcal{H})$. It also has an interesting interpretation in terms of a system of classical identical particles 20.

Global rotations and translations act as

$$
\left|\alpha_{i}\right\rangle \rightarrow\left|\mathrm{e}^{\mathrm{i} \vartheta} \alpha_{i}\right\rangle=\mathrm{e}^{\mathrm{i} \vartheta a^{\dagger} a}\left|\alpha_{i}\right\rangle \quad \text { and } \quad\left|\alpha_{i}\right\rangle \rightarrow\left|\alpha_{i}+\beta\right\rangle=\mathrm{e}^{\beta a^{\dagger}}\left|\alpha_{i}\right\rangle,
$$

respectively, and shift the Kähler potential by a gauge transformation,

$$
K \rightarrow K+8 \pi \theta \sum_{i=1}^{k}\left(\bar{\beta} \alpha_{i}+\beta \bar{\alpha}_{i}+\beta \bar{\beta}\right),
$$

leaving the metric unchanged. Furthermore, (3.23) is invariant under permutations of the $\alpha_{i}$. When passing to center-of-mass and barycentric coordinates

$$
s=\frac{1}{k} \sum_{i=1}^{k} \alpha_{i} \quad \text { and } \quad w_{i}=\alpha_{i}-s \quad \text { such that } \quad \sum_{i=1}^{k} w_{i}=0 \quad,
$$

we get the decomposition

$$
K=8 \pi \theta k|s|^{2}+8 \pi \theta \ln \operatorname{det}\left(\mathrm{e}^{\bar{w}_{i} w_{j}}\right),
$$

which shows that the metric depends only on difference coordinates $\alpha_{i}-\alpha_{j}$. One may also extract the diagonal (free) part via

$$
K=8 \pi \theta \sum_{i=1}^{k}\left|\alpha_{i}\right|^{2}+8 \pi \theta \ln \operatorname{det}\left(\mathrm{e}^{-\frac{1}{2}\left|\alpha_{i}-\alpha_{j}\right|^{2}+\frac{1}{2}\left(\bar{\alpha}_{i} \alpha_{j}-\bar{\alpha}_{j} \alpha_{i}\right)}\right) .
$$

From this expression it is easy to see a cluster decomposition property: Upon splitting the moduli into two groups, $\left\{\alpha_{i}\right\}=\left\{\alpha_{\ell}^{\prime}, \alpha_{m}^{\prime \prime}\right\}$, and separating these to infinity,

$$
\lim _{\left|\alpha_{\ell}^{\prime}-\alpha_{m}^{\prime \prime}\right| \rightarrow \infty} K\left(\left\{\alpha_{i}\right\}\right)=K\left(\left\{\alpha_{\ell}^{\prime}\right\}\right)+K\left(\left\{\alpha_{m}^{\prime \prime}\right\}\right) .
$$

In particular, an isolated single lump at $\alpha_{q}$ asymptotically contributes with $\left|\alpha_{q}\right|^{2}$ to $K$. Therefore, the moduli-space metric becomes flat for large mutual separations, $\left|\alpha_{i}-\alpha_{j}\right| \rightarrow \infty$.

More interesting is the limit of coinciding lumps, say $\alpha_{i} \rightarrow \alpha$ for $i=q_{1}, \ldots, q_{r}$. Some lengthy algebra shows that then

$$
K \rightarrow 8 \pi \theta \sum_{q_{\ell}>q_{m}} \ln \left|\alpha_{q_{\ell}}-\alpha_{q_{m}}\right|^{2}+K^{\prime} \quad \text { where still } \quad K^{\prime}=\ln \operatorname{det}(\langle T \mid T\rangle),
$$

but after making inside $|T\rangle=\left(\left|\alpha_{1}\right\rangle, \ldots,\left|\alpha_{k}\right\rangle\right)$ the replacement

$$
\left\{\left|\alpha_{q_{1}}\right\rangle,\left|\alpha_{q_{2}}\right\rangle, \ldots,\left|\alpha_{q_{r}}\right\rangle\right\} \rightarrow\left\{|\alpha\rangle, a^{\dagger}|\alpha\rangle, \ldots,\left(a^{\dagger}\right)^{r-1}|\alpha\rangle\right\} .
$$

The coordinate singularity in (3.30) can be removed by passing to new coordinates, namely elementary symmetric polynomials in $\alpha_{q_{\ell}}-\alpha$, which correspond precisely to the new states in (3.31). $K^{\prime}$ produces the same metric as $K$ but is smooth at the coincidence locus. In the most general situation, $|T\rangle$ is composed of various blocks like in (3.31), of different sizes $r$, but the formula for the smooth Kähler potential $K^{\prime}$ in (3.30) remains correct. 


\section{Moduli-space trajectories for two-soliton scattering}

For concreteness, let us display the simplest nontrivial case, i.e. $k=2$. The moduli space $\mathcal{M}_{2}$ of rank-two BPS projectors is parametrized by $\{\alpha, \beta\} \simeq\{\beta, \alpha\} \in \mathbb{C}^{2} / S_{2}$. Since the details have been given in [16], we can be short here.

The static two-lump configuration is derived from (2.25) as

$$
\phi_{\alpha \beta}=\mathbb{1}-2 P_{\alpha \beta}=\mathbb{1}-2 \frac{|\alpha\rangle\langle\beta \mid \beta\rangle\langle\alpha|+| \beta\rangle\langle\alpha \mid \alpha\rangle\langle\beta|-| \alpha\rangle\langle\alpha \mid \beta\rangle\langle\beta|-| \beta\rangle\langle\beta \mid \alpha\rangle\langle\alpha|}{\langle\alpha \mid \alpha\rangle\langle\beta \mid \beta\rangle-\langle\alpha \mid \beta\rangle\langle\beta \mid \alpha\rangle} .
$$

Writing $\alpha=s+w$ and $\beta=s-w$ as well as $2 w=: r \mathrm{e}^{\mathrm{i} \varphi}$, the corresponding Kähler potential reads

$$
\begin{aligned}
K & =8 \pi \theta \ln \left(\mathrm{e}^{\alpha \bar{\alpha}+\beta \bar{\beta}}-\mathrm{e}^{\alpha \bar{\beta}+\beta \bar{\alpha}}\right)=8 \pi \theta\left[2 s \bar{s}+2 w \bar{w}+\ln \left(1-\mathrm{e}^{-4 w \bar{w}}\right)\right] \\
& =8 \pi \theta \ln \left(2 \mathrm{e}^{2 s \bar{s}} \sinh \frac{r^{2}}{2}\right) \quad=8 \pi \theta\left[2 s \bar{s}+\frac{1}{2} r^{2}+\ln \left(1-\mathrm{e}^{-r^{2}}\right)\right]
\end{aligned}
$$

with the limits

$$
K=8 \pi \theta\left[2 s \bar{s}+\frac{1}{2} r^{2}-\mathrm{e}^{-r^{2}}+O\left(\mathrm{e}^{-2 r^{2}}\right)\right] \quad \text { and } \quad K=8 \pi \theta\left[2 s \bar{s}+\ln r^{2}+\frac{1}{24} r^{4}+O\left(r^{8}\right)\right] .
$$

It yields the metric

$$
\mathrm{d} \ell^{2}=16 \pi \theta[\mathrm{d} s \mathrm{~d} \bar{s}+\Omega \mathrm{d} w \mathrm{~d} \bar{w}]=4 \pi \theta\left[4 \mathrm{~d} s \mathrm{~d} \bar{s}+\Omega\left(r^{2}\right)\left(\mathrm{d} r^{2}+r^{2} \mathrm{~d} \varphi^{2}\right)\right]
$$

with the conformal factor

$$
\Omega\left(r^{2}\right)=\frac{1}{4 \pi \theta} \partial_{r^{2}}\left(r^{2} \partial_{r^{2}} K\right)=\frac{1-2 r^{2} \mathrm{e}^{-r^{2}}-\mathrm{e}^{-2 r^{2}}}{\left(1-\mathrm{e}^{-r^{2}}\right)^{2}}=\frac{\sinh r^{2}-r^{2}}{\cosh r^{2}-1},
$$

possessing the limits

$$
\Omega\left(r^{2}\right)=1+\left(1-2 r^{2}\right) \mathrm{e}^{-r^{2}}+O\left(\mathrm{e}^{-2 r^{2}}\right) \quad \text { and } \quad \Omega\left(r^{2}\right)=\frac{1}{3} r^{2}-\frac{1}{90} r^{6}+O\left(r^{10}\right) .
$$

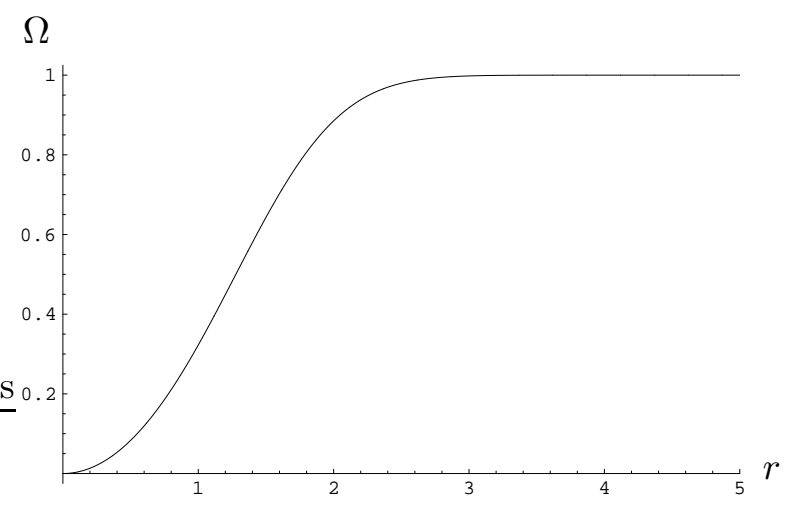

Clearly, the metric becomes flat for $r \rightarrow \infty$ but develops a conical singularity with an angle of $4 \pi$ at $r=0$. The latter is removed by passing to the symmetric coordinate $\sigma=w^{2}$, in terms of which one finds

$$
\mathrm{d} \ell^{2}=16 \pi \theta\left[\mathrm{d} s \mathrm{~d} \bar{s}+\frac{\Omega\left(r^{2} \rightarrow 4 \sqrt{\sigma \bar{\sigma}}\right)}{4 \sqrt{\sigma \bar{\sigma}}} \mathrm{d} \sigma \mathrm{d} \bar{\sigma}\right]=16 \pi \theta\left[\mathrm{d} s \mathrm{~d} \bar{s}+\left(\frac{1}{3}-\frac{8}{45} \sigma \bar{\sigma}+O\left((\sigma \bar{\sigma})^{2}\right)\right) \mathrm{d} \sigma \mathrm{d} \bar{\sigma}\right] .
$$


Due to the decoupling of the trivial center-of-mass dynamics, $\mathcal{M}_{2}=\mathbb{C} \times \mathcal{M}_{\text {rel }}$, with $\mathcal{M}_{\text {rel }} \simeq \mathbb{C}$ rotationally symmetric, asymptotically flat, and of positive curvature $R=\frac{1}{4 \pi \theta}\left[\frac{5}{4}-\frac{6}{175} r^{4}+O\left(r^{8}\right)\right]$. Head-on scattering of two lumps corresponds to a single radial trajectory in $\mathcal{M}_{\text {rel }}$, which in the smooth coordinate $\sigma$ must pass straight through the origin. In the 'doubled coordinate' $w=\sqrt{\sigma}$, we then see two straight trajectories with $90^{\circ}$ scattering off the singularity in the Moyal plane.

This picture persists for the scattering of two composite lumps, i.e. lumps obtained by fusing

$$
\alpha_{i} \rightarrow \alpha \text { for } i=1, \ldots, r_{1} \quad \text { and } \quad \alpha_{r_{1}+j} \rightarrow \beta \text { for } j=1, \ldots, r_{2} \text {. }
$$

The decoupling of the center-of-mass coordinate (now for $k=r_{1}+r_{2}$ ) is achieved by writing

$$
\alpha=s+r_{2} w \quad \text { and } \quad \beta=s-r_{1} w \quad \text { such that } \quad \alpha-\beta=\left(r_{1}+r_{2}\right) w=: r \mathrm{e}^{\mathrm{i} \varphi} \text {, }
$$

and one obtains

$$
\begin{gathered}
K \quad=8 \pi \theta\left[\left(r_{1}+r_{2}\right) s \bar{s}+\frac{r_{1} r_{2}}{r_{1}+r_{2}} r^{2}+\ln \left(1-\mathcal{P} \mathrm{e}^{-r^{2}}+O\left(\mathrm{e}^{-2 r^{2}}\right)\right)\right] \\
\stackrel{r \rightarrow 0}{\longrightarrow} 8 \pi \theta\left[\left(r_{1}+r_{2}\right) s \bar{s}+c_{0}+c_{1} \ln r^{2}+c_{2} r^{4}+O\left(r^{6}\right)\right]
\end{gathered}
$$

where $\mathcal{P}$ is a polynomial in $r^{2}$ and $c_{0}, c_{1}$ and $c_{2}$ are constants. As in (4.3), the absence of the $r^{2}$ term (and $c_{2} \neq 0$ ) leads to a conformal factor $\Omega \sim r^{2}$ for $r \rightarrow 0$ and the same conical singularity for any value of $r_{1}$ or $r_{2}$. Its remedy by employing the coordinate $\sigma=w^{2}$ demonstrates that the $90^{\circ}$ scattering angle is universal for head-on motion. Only for more special situations with simultaneous head-on collision of $k(>2)$ solitons one will get $\frac{\pi}{k}$ scattering.

Let us return to the simple case of $r_{1}=r_{2}=1$ and drop the center-of mass coordinate. The motion in $\mathcal{M}_{\text {rel }}$ is geodesic with conformal factor $\Omega\left(r^{2}\right)$ given in (4.5). It conserves angular momentum and energy,

$$
l=\Omega r^{2} \dot{\phi}=v_{\infty} b \quad \text { and } \quad e=\frac{1}{2} \Omega \dot{r}^{2}+\frac{l^{2}}{2 \Omega r^{2}}=\frac{1}{2} v_{\infty}^{2} \quad,
$$

respectively, with the asymptotic speed $v_{\infty}$ and the impact parameter

$$
b=l / \sqrt{2 e}=r_{\min } \sqrt{\Omega\left(r_{\min }\right)} .
$$

Hence, the trajectory is given by

$$
\frac{\mathrm{d} r}{\mathrm{~d} \phi}=\frac{r^{2}}{b} \sqrt{\Omega-b^{2} / r^{2}}
$$

and we obtain the scattering angle

$$
\Theta(b)=\pi-2 \int_{r_{\min }}^{\infty} \frac{b \mathrm{~d} r}{r^{2} \sqrt{\Omega-b^{2} / r^{2}}},
$$

which varies between 0 (for $b \rightarrow \infty$ and $\Omega \rightarrow 1$ ) and $\frac{\pi}{2}$ (for $b \rightarrow 0$ and $\Omega \rightarrow \frac{1}{3} r^{2}$ ). Therefore, if we fix $b$ and vary $v_{\infty}$, the trajectory is unchanged. The total energy of the $k=2$ system is

$$
E_{\text {mod }}:=E\left[\phi_{\alpha}\right]=16 \pi+8 \pi \theta e=16 \pi+4 \pi \theta v_{\infty}^{2}
$$


Good agreement with the full field-theory dynamics is expected only for small values of $v_{\infty}$. For the cases $r_{1}=1$ (triangles), 2(diamonds), 4(boxes) and $r_{2}=1$ the function $\Theta(b)$ is plotted below.

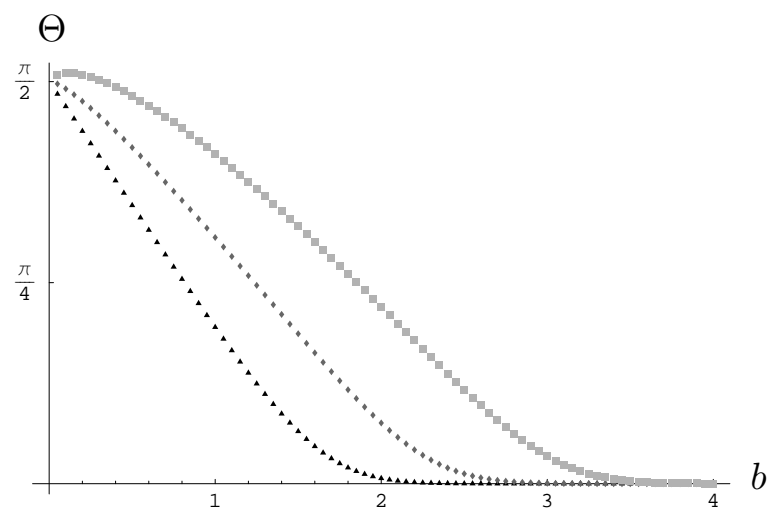

\section{Comparison with time-dependent field-theory solutions}

According to the general arguments about the adiabatic approximation, the moduli-space dynamics described in the previous section should apply to the whole family $S_{\gamma}$ of actions in (3.3). To test the quality of the approach, one would like to compare the moduli-space scattering trajectories with the time evolution of the energy-density maxima of the corresponding classical field configurations. Since widely separated lumps roam essentially independently of each other, we already know the large-time asymptotics: a (multiplicative) superposition of several one-soliton configurations of the form $\phi_{\alpha}=\mathbb{1}-2 P_{\alpha}$, after applying individual translations and boosts.

To simplify the discussion, let us consider just two lumps of rank one each, i.e. combine two copies of $\phi_{0}=\mathbb{1}-2|0\rangle\langle 0|$. For large (positive or negative) times we then must have

$$
\begin{aligned}
\phi(t \rightarrow \pm \infty) & \simeq\left(\mathbb{1}-\left(1-\mathrm{e}^{\mathrm{i} \delta_{1}}\right) U_{1}|0\rangle\langle 0| U_{1}^{\dagger}\right)\left(\mathbb{1}-\left(1-\mathrm{e}^{\mathrm{i} \delta_{2}}\right) U_{2}|0\rangle\langle 0| U_{2}^{\dagger}\right) \\
& \simeq \mathbb{1}-\left(1-\mathrm{e}^{\mathrm{i} \delta_{1}}\right) U_{1}|0\rangle\left\langle 0\left|U_{1}^{\dagger}-\left(1-\mathrm{e}^{\mathrm{i} \delta_{2}}\right) U_{2}\right| 0\right\rangle\langle 0| U_{2}^{\dagger},
\end{aligned}
$$

where

$$
U_{i}=U\left(\vec{v}_{i}^{ \pm}, \vec{r}_{i}^{ \pm}, t\right) \quad \text { for } \quad i=1,2 \quad \text { and } \quad t \rightarrow \pm \infty
$$

are unitary transformations implementing translations by $\vec{r}_{i}^{ \pm}$and boosts with velocities $\vec{v}_{i}^{ \pm}$in the Moyal plane. Note that (time-dependent) solitons need no longer be Grassmannian, and so we must allow in (5.1) for the slightly more general prefactors with velocity-dependent phases $\delta_{i}$ [11, 22]. If the scattering angle differs from $\pi$, i.e. if nontrivial scattering occurs, then the late velocities $\vec{v}_{i}^{+}$ must differ from the early ones $\vec{v}_{i}^{-}$.

Outside the value $\gamma=1$, the solitons are affected by each other's presence, and no integrability protects them from shrinking and decay. Yet, in cases where their lifetime is sufficiently long the configurations (5.1) can still be approached for not too large times, and scattering data are viable. In the absence of exact time-dependent solutions, however, numerical investigations are needed for confirmation. To the author's knowledge, computer analysis has been applied only in the commutative case $(\theta=0)$ for $\gamma=0$ and $\mathcal{G}=\mathrm{O}(3)$, where it established the universality of 
$90^{\circ}$ head-on two-soliton scattering [21]. In the noncommutative realm, the equation of motion for $\mathcal{G}=\mathrm{U}(1)$ and $\gamma=0$ to solve in the operator formulation is

$$
\begin{aligned}
0 & =\theta \partial_{t}\left(\phi^{\dagger} \partial_{t} \phi\right)+\left[a, \phi^{\dagger}\left[a^{\dagger}, \phi\right]\right]+\left[a^{\dagger}, \phi^{\dagger}[a, \phi]\right] \\
& =\theta \partial_{t}\left(\phi^{\dagger} \partial_{t} \phi\right)+\phi^{\dagger}\left[a,\left[a^{\dagger}, \phi\right]\right]-\left[a,\left[a^{\dagger}, \phi^{\dagger}\right]\right] \phi .
\end{aligned}
$$

With $\phi \in \mathrm{U}(\mathcal{H})$ viewed as an infinite-size matrix $\left(\phi_{m n}\right)$ in the Fock-space basis (2.20), it reads

$$
\begin{aligned}
\theta \partial_{t}\left(\phi_{n m}^{*} \partial_{t} \phi_{n \ell}\right)=(m-\ell) \phi_{n m}^{*} \phi_{n \ell} & +\sqrt{(n+1)(\ell+1)} \phi_{n m}^{*} \phi_{n+1 \ell+1}+\sqrt{n \ell} \phi_{n m}^{*} \phi_{n-1 \ell-1} \\
& -\sqrt{(n+1)(m+1)} \phi_{n+1 m+1}^{*} \phi_{n \ell}-\sqrt{n m} \phi_{n-1 m-1}^{*} \phi_{n \ell} .
\end{aligned}
$$

It would be interesting to analyze this coupled initial-value problem numerically.

For $\gamma=1$, the situation is entirely different since exact multi-soliton solutions are available [11. 22, 23. As a warm-up, consider the generic one-soliton configuration,

$$
\phi_{1}(t)=(\mathbb{1}-\widetilde{P}(t))+\frac{\mu}{\bar{\mu}} \widetilde{P}(t)=\mathbb{1}-\left(1-\frac{\mu}{\bar{\mu}}\right) \widetilde{P}(t) \quad \text { with } \quad \widetilde{P}(t)=U(\mu, t) P_{\alpha} U(\mu, t)^{\dagger}
$$

where $U(\mu, t)$ is the unitary transformation effecting a boost with velocity

$$
\vec{v} \equiv\left(v_{x}, v_{y}\right)=-\left(\frac{\mu+\bar{\mu}}{\mu \bar{\mu}+1}, \frac{\mu \bar{\mu}-1}{\mu \bar{\mu}+1}\right) \quad \Longleftrightarrow \quad \mu=-\frac{v_{x}+\mathrm{i} \sqrt{1-\vec{v}^{2}}}{1-v_{y}} \in \mathbb{C} \backslash \mathbb{R} .
$$

The energy of this configuration is found to be

$$
E\left[\phi_{1}\right] \equiv E(\vec{v})=8 \pi \frac{\sqrt{1-\vec{v}^{2}}}{1-v_{y}^{2}}=8 \pi\left(1-\frac{1}{2} v_{x}^{2}+\frac{1}{2} v_{y}^{2}+\ldots\right)
$$

The exact two-soliton solution of rank two in the Ward model reads [1]

$$
\phi_{2}(t)=\mathbb{1}-\frac{\left(1-\frac{\mu_{1}}{\bar{\mu}_{1}}\right)|1\rangle\langle 2 \mid 2\rangle\left\langle 1\left|+\left(1-\frac{\mu_{2}}{\bar{\mu}_{2}}\right)\right| 2\right\rangle\langle 1 \mid 1\rangle\left\langle 2\left|-\mu\left(1-\frac{\mu_{2}}{\bar{\mu}_{1}}\right)\right| 1\right\rangle\langle 1 \mid 2\rangle\left\langle 2\left|-\mu\left(1-\frac{\mu_{1}}{\bar{\mu}_{2}}\right)\right| 2\right\rangle\langle 2 \mid 1\rangle\langle 1|}{\langle 1 \mid 1\rangle\langle 2 \mid 2\rangle-\mu\langle 1 \mid 2\rangle\langle 2 \mid 1\rangle}
$$

with

$$
\mu=\frac{\left(\mu_{1}-\bar{\mu}_{1}\right)\left(\mu_{2}-\bar{\mu}_{2}\right)}{\left(\mu_{1}-\bar{\mu}_{2}\right)\left(\mu_{2}-\bar{\mu}_{1}\right)} \quad \text { and } \quad|i\rangle=U\left(\mu_{i}, t\right)\left|\alpha_{i}\right\rangle \quad \text { for } \quad i=1,2 .
$$

Here, $\mu_{i}$ parametrize the (constant) velocities of the two solitons like in (5.6), and $\alpha_{i}$ are their positions at $t=0$. The unitary transformations $U$ boost the vacuum state, and so the time-dependent states $|i\rangle$ are just moving-frame vacua for the two lumps. It is easy to verify that in the static limit $\mu_{i} \rightarrow-\mathrm{i}$ the configuration (5.8) tends to the static solution (4.1) as long as $\alpha_{1} \neq \alpha_{2}$. For large times, the overlap $\langle 1 \mid 2\rangle$ dies away, and indeed the form (5.1) is attained. However, we see that $\vec{v}_{i}^{+}=\vec{v}_{i}^{-}=\vec{v}_{i}$ since the velocities do not change, and thus there is no scattering! This is also evinced by a no-force property of Ward solitons, borne out by their energy additivity:

$$
E\left[\phi_{2}\right]=E\left(\vec{v}_{1}\right)+E\left(\vec{v}_{2}\right)
$$

Even for small velocities, the energy density of the solution (5.8) does not follow the modulispace dynamics of the previous section (except of course at very large impact parameter where the 
scattering disappears). Therefore, we should look for other classes of exact two-soliton solutions. Recently it has been established 24] for the commutative case that all Ward model multi-solitons are obtained from the one-soliton configurations (5.5) by dressing and fusing operations. ${ }^{6}$ The energy is additive under dressing and unchanged under fusing. In fact, (5.8) was constructed by dressing (5.5) with a copy.

For comparison with the $k=2$ case of the previous section, it remains to consider fusing the two-soliton (5.8). This is achieved by putting $\alpha_{1}=\alpha_{2}=\alpha$ and sending both velocities to zero. ${ }^{7}$ In this limit a new type of time dependence emerges. Putting in (5.8)

$$
\mu_{1}=-\mathrm{i}+\epsilon \quad \text { and } \quad \mu_{2}=-\mathrm{i}-\epsilon \quad \text { with } \quad \mathbb{C} \ni \epsilon \rightarrow 0
$$

and observing that

$$
U(-\mathrm{i} \pm \epsilon, t)|\alpha\rangle=\mathrm{e}^{-|\epsilon|^{2} t^{2} / 4 \theta} \mathrm{e}^{ \pm \alpha \epsilon t / \sqrt{2 \theta}}\left(1 \mp \frac{\bar{\epsilon} t}{\sqrt{2 \theta}} a^{\dagger}+O\left(\epsilon^{2}\right)\right)|\alpha\rangle,
$$

we learn that any time dependence comes in the combination of $\epsilon t / \sqrt{2 \theta}$. It is crucial to observe that the limits $\epsilon \rightarrow 0$ and $|t| \rightarrow \infty$ do not commute, and so the asymptotic behavior of (5.8) is modified under fusing. The result is

$$
\widetilde{\phi}_{2}(t):=\lim _{\epsilon \rightarrow 0} \phi_{2}(t)=\left(\mathbb{1}-2 \frac{|\alpha\rangle\langle\alpha|}{\langle\alpha \mid \alpha\rangle}\right)\left(\mathbb{1}-2 \frac{|\widetilde{\alpha}\rangle\langle\widetilde{\alpha}|}{\langle\widetilde{\alpha} \mid \widetilde{\alpha}\rangle}\right),
$$

where the time dependence hides in

$$
|\widetilde{\alpha}\rangle=|\alpha\rangle-\text { i } t \sqrt{\frac{2}{\theta}}\left|\alpha^{\perp}\right\rangle \quad \text { with } \quad\left|\alpha^{\perp}\right\rangle=\left(a^{\dagger}-\bar{\alpha}\right)|\alpha\rangle
$$

being orthogonal to $|\alpha\rangle$. More explicitly,

$$
\widetilde{\phi}_{2}(t)=\mathbb{1}-\frac{2}{\theta+2 t^{2}}\left\{2 t^{2}\left(|\alpha\rangle\left\langle\alpha|+| \alpha^{\perp}\right\rangle\left\langle\alpha^{\perp}\right|\right)-\mathrm{i} t \sqrt{2 \theta}\left(|\alpha\rangle\left\langle\alpha^{\perp}|+| \alpha^{\perp}\right\rangle\langle\alpha|\right)\right\},
$$

which at $t=0$ momentarily degenerates to $\phi=\mathbb{1}$.

This solution can also be constructed directly by the dressing method, starting from the ansatz (5.13) with an unknown state $|\widetilde{\alpha}\rangle$. In this way one arrives at the conditions [22]

$$
a|\widetilde{\alpha}\rangle+\left[a, \frac{|\alpha\rangle\langle\alpha|}{\langle\alpha \mid \alpha\rangle}\right]|\widetilde{\alpha}\rangle=|\widetilde{\alpha}\rangle Z_{1} \quad \text { and } \quad \partial_{t}|\widetilde{\alpha}\rangle+\mathrm{i} \sqrt{\frac{2}{\theta}}\left[a^{\dagger}, \frac{|\alpha\rangle\langle\alpha|}{\langle\alpha \mid \alpha\rangle}\right]|\widetilde{\alpha}\rangle=|\widetilde{\alpha}\rangle Z_{2},
$$

where $Z_{1}$ and $Z_{2}$ are functions of $t$ to be determined. We read off that $Z_{1}=\alpha$ and fix the (inessential) normalization such that $Z_{2}=0$. It is not hard then to recover (5.14) as the general solution indeed.

Putting $\alpha=0$ for simplicity, the energy density of (5.15) is readily computed to be 22$]$

$$
\begin{array}{r}
\mathcal{E}=\frac{4 \theta}{\left(\theta+2 t^{2}\right)^{2}}\left\{(|0\rangle\langle 0|+| 1\rangle\langle 1|)+\frac{2 t^{2}}{\theta}(2|0\rangle\langle 0|+| 1\rangle\langle 1|+| 2\rangle\langle 2|)+\frac{4 t^{4}}{\theta^{2}}(|1\rangle\langle 1|+| 2\rangle\langle 2|) \quad(5.17)\right. \\
\left.\quad-\frac{2 t^{2}}{\theta}\left(\frac{1}{\sqrt{2}}|2\rangle\left\langle 0\left|+\frac{1}{\sqrt{2}}\right| 0\right\rangle\langle 2|\right)-\mathrm{i} \frac{2^{3 / 2} t^{3}}{\theta^{3 / 2}}\left(|1\rangle\langle 0|-| 0\rangle\left\langle 1\left|+\frac{1}{\sqrt{2}}\right| 2\right\rangle\left\langle 1\left|-\frac{1}{\sqrt{2}}\right| 1\right\rangle\langle 2|\right)\right\},
\end{array}
$$

\footnotetext{
${ }^{6}$ This result presumably extends to the noncommutative case.

${ }^{7}$ The more general situation of merely equal velocities is related by boosting the center of mass.
} 
with $E\left[\widetilde{\phi}_{2}\right]=2 \pi \theta \operatorname{tr} \mathcal{E}=16 \pi$ as should be. Matching with $E_{\bmod }$ in (4.16) enforces $v_{\infty}=0$ which, however, does not restrict $b$ in any way. Employing the Moyal-Weyl correspondence, the energy density in the Moyal plane takes the form [22]

$\mathcal{E}_{\star}=\frac{16 \mathrm{e}^{-r^{2} / \theta}}{\theta\left(1+2 t^{2} / \theta\right)^{2}}\left\{\frac{r^{2}}{\theta}+\left(1-\frac{r^{2}}{\theta}+\frac{r^{4}}{\theta^{2}}\right) \frac{2 t^{2}}{\theta}+\left(-\frac{r^{2}}{\theta}+\frac{r^{4}}{\theta^{2}}\right) \frac{4 t^{4}}{\theta^{2}}-\left(\frac{x^{2}}{\theta}-\frac{y^{2}}{\theta}\right) \frac{2 t^{2}}{\theta}-\frac{4 y r^{2} t^{3}}{\theta^{3}}\right\}$.

Unfortunately, this energy distribution is invariant under spacetime inversion and has a ring-like structure in the Moyal plane, localized at the origin like a bound state. Hence, we do not find any scattering solutions with $k=2$ in the noncommutative $\mathrm{U}(1)$ Ward model. ${ }^{8}$

Interestingly, the nonabelian Ward model is very different in this regard because of its larger moduli space: Fusing the U(2) two-soliton solution also features ring-like configurations but also admits moduli choices which produce genuine $90^{\circ}$ scattering, in the commutative [25, 26, 27, 8] as well as in the noncommutative [22] case. The corresponding moduli-space approximation was recently considered in [9] and [28, respectively. For $\theta=0$, it seems to agree with the analytical and numerical field-theory results obtained earlier.

In summary, their moduli-space motion does not approximate the extended abelian sigmamodel soliton scattering in the Moyal plane equally well for all values of the family parameter $\gamma$. Numerical analysis is needed to make the case for $\gamma<1$. For the integrable value $\gamma=1$ (the abelian Ward model), however, we are curiously lacking the field-theory dynamics which the moduli-space kinematics is supposed to mimic. ${ }^{9}$ It is therefore conceivable that in this case, even for arbitrary small velocities, the soliton scattering takes place far away from their moduli space, if it occurs at all! Certainly, the known no-scattering multi-solitons are not seen in the moduli space, which challenges Manton's paradigm. In part responsible for this failure seems to be the absence of magnetic forcing in the moduli space in contrast to the crucial importance of the WZW-like action term for integrability. Perhaps a numerical study can help to answer this conundrum.

\section{Acknowledgements}

The authors are grateful to Andrei V. Domrin and Alexander D. Popov for discussions and reading the manuscript. This work was partially supported by the Deutsche Forschungsgemeinschaft.

\section{References}

[1] N.S. Manton, A remark on the scattering of BPS monopoles, Phys. Lett. B 110 (1982) 54.

[2] N.S. Manton and P. Sutcliffe, Topological solitons, Cambridge University Press (2004).

[3] K. Uhlenbeck, Harmonic maps into Lie groups: classical solutions of the chiral model, J. Diff. Geom. 30 (1989) 1-50.

[4] J.C. Wood, Explicit construction and parameterization of harmonic two spheres in the unitary group, Proc. London Math. Soc. 58 (1989) 608.

[5] W.J. Zakrzewski, Low dimensional sigma models, Hilger (1989).

\footnotetext{
${ }^{8}$ We generalized the ansatz (5.13) by relaxing the first projector, allowing for higher-rank projectors and admitting time-dependent coefficients, all without success.

${ }^{9}$ This also applies to the no-scattering solutions of the nonabelian models.
} 
[6] R.S. Ward, Soliton solutions in an integrable chiral model in $2+1$ dimensions, J. Math. Phys. 29 (1988) 386.

[7] R.S. Ward, Classical solutions of the chiral model, unitons, and holomorphic vector bundles, Commun. Math. Phys. 128 (1990) 319.

[8] T.A. Ioannidou and W.J. Zakrzewski, Solutions of the modified chiral model in 2+1 dimensions, J. Math. Phys. 39 (1998) 2693 hep-th/9802122.

[9] M. Dunajski and N.S. Manton, Reduced dynamics of Ward solitons, Nonlinearity 18 (2005) 1677 hep-th/0411068.

[10] J.A. Harvey, Komaba lectures on noncommutative solitons and D-branes, hep-th/0102076

[11] O. Lechtenfeld and A.D. Popov, Noncommutative multi-solitons in $2+1$ dimensions, JHEP 0111 (2001) 040 hep-th/0106213.

[12] A.V. Domrin, O. Lechtenfeld and S. Petersen, Sigma-model solitons in the noncommutative plane: Construction and stability analysis, JHEP 0503 (2005) 045 hep-th/0412001.

[13] O. Lechtenfeld, A.D. Popov and B. Spendig, Noncommutative solitons in open N=2 string theory, JHEP 0106 (2001) 011 hep-th/0103196.

[14] R. Gopakumar, S. Minwalla and A. Strominger, Noncommutative solitons, JHEP 0005 (2000) 020 hep-th/0003160.

[15] M. Aganagic, R. Gopakumar, S. Minwalla and A. Strominger, Unstable solitons in noncommutative gauge theory, JHEP 0104 (2001) 001 hep-th/0009142.

[16] U. Lindström, M. Roček and R. von Unge, Non-commutative soliton scattering, JHEP 0012 (2000) 004 hep-th/0008108.

[17] R. Gopakumar, M. Headrick and M. Spradlin, On noncommutative multi-solitons, Commun. Math. Phys. 233 (2003) 355 hep-th/0103256.

[18] L. Hadasz, U. Lindström, M. Roček and R. von Unge, Noncommutative multisolitons: Moduli spaces, quantization, finite theta effects and stability, JHEP 0106 (2001) 040 hep-th/0104017.

[19] M. Hamanaka, Y. Imaizumi and N. Ohta, Moduli space and scattering of D0-branes in noncommutative super Yang-Mills theory, Phys. Lett. B 529 (2002) 163 hep-th/0112050.

[20] T.H. Hansson, S.B. Isakov, J.M. Leinaas, U. Lindström, Classical phase space and statistical mechanics of identical particles, quant-ph/0003121; Exclusion statistics in classical mechanics, quant-ph/0004108.

[21] W.J. Zakrzewski, Soliton-like scattering in the $O(3)$ sigma model in $2+1$ dimensions, Nonlinearity 4 (1991) 429-475.

[22] O. Lechtenfeld and A.D. Popov, Scattering of noncommutative solitons in $2+1$ dimensions, Phys. Lett. B 523 (2001) 178 hep-th/0108118.

[23] M. Wolf, Soliton antisoliton scattering configurations in a noncommutative sigma model in 2+1 dimensions, JHEP 0206 (2002) 055 hep-th/0204185.

[24] B. Dai, C.L. Terng and K. Uhlenbeck, On the space-time monopole equation, math.dg/0602607

[25] R. S. Ward, Nontrivial scattering of localized solitons in a (2+1)-dimensional integrable system, Phys. Lett. A 208 (1995) 203.

[26] T.A. Ioannidou, Soliton solutions and nontrivial scattering in an integrable chiral model in 2+1 dimensions, J. Math. Phys. 37 (1996) 3422 hep-th/9604126.

[27] C. Anand, Ward's solitons, Geom. Topol. 1 (1997) 9.

[28] K. Furuta, T. Inami, H. Nakajima and M. Yamamoto, Low-energy dynamics of noncommutative CP(1) solitons in 2+1 dimensions, Phys. Lett. B 537 (2002) 165 hep-th/0203125. 